\title{
Editorial: New Advances in Electrocochleography for Clinical and Basic Investigation
}

\author{
Martin Pienkowski ${ }^{1 *}$, Oliver F. Adunka ${ }^{2}$ and Jeffery T. Lichtenhan ${ }^{3 *}$ \\ ${ }^{1}$ Salus University, Elkins Park, PA, United States, ${ }^{2}$ Wexner Medical Center, The Ohio State University, Columbus, OH, \\ United States, ${ }^{3}$ School of Medicine, Washington University in St. Louis, St. Louis, MO, United States
}

Keywords: electrocochleography (ECochG), cochlea, hearing disorders, balance disorders, cochlear implants

\section{Editorial on the Research Topic}

\section{New Advances in Electrocochleography for Clinical and Basic Investigation}

\section{OPEN ACCESS}

Edited and reviewed by: Gavin M. Bidelman University of Memphis, United States

${ }^{*}$ Correspondence: Martin Pienkowski mpienkowski@salus.edu Jeffery T. Lichtenhan jlichtenhan@wustl.edu

Specialty section:

This article was submitted to Auditory Cognitive Neuroscience, a section of the journal Frontiers in Neuroscience

Received: 05 April 2018 Accepted: 20 April 2018 Published: 08 May 2018

Citation:

Pienkowski M, Adunka OF and Lichtenhan JT (2018) Editorial: New Advances in Electrocochleography for Clinical and Basic Investigation.

Front. Neurosci. 12:310. doi: 10.3389/fnins.2018.00310
Electrocochleography (ECochG) is a technique for recording evoked potentials from the inner ear, generally believed to originate from hair cells and nerve fibers. It is useful for assessing inner ear function in both laboratory and clinical settings. The abbreviation ECochG is preferable to ECoG, because the latter can be confused with "electrocorticogram" (Ferraro, 1986). ECochG measurements are typically made from the ear canal or eardrum (extratympanic), from the promontory or round window niche (transtympanic), or from inside the cochlea (intracochlear). Extratympanic ECochG recordings are most commonly made with "tiptrodes" (gold foil wrapped around insert earphones) or "tymptrodes" (electrodes placed directly on the tympanic membrane). While the amplitude of tymptrode measurements can be up to an order of magnitude larger than tiptrode measurements (Ferraro and Ferguson, 1989), transtympanic amplitudes can be far more than an order of magnitude larger than those on the eardrum (e.g., Ruth et al., 1988). We thus suggest that extratympanic measurements are best classified as far-field, and transtympanic measurements as near-field.

We will give a brief overview of ECochG before reviewing its traditional uses, and surveying recent advances that promise new applications in the assessment of auditory and vestibular function. References to the 23 papers collected for this Research Topic have been hyperlinked to Frontiers webpages. A more extensive historical overview of ECochG, including its basic features and applications, was provided by Eggermont. A complementary review by Gibson offers tips for optimizing ECochG recordings in different clinical situations. Electrovestibulography (EVestG) is an analogous emerging technique for characterizing vestibular hair cell and nerve function, and was reviewed by Brown et al.

Sensory cells of the inner ear can be manipulated, damaged, or destroyed in varying degrees depending on the ototoxic agent, administration approach, and dose, giving rise to hearing deficits at specific sound frequencies and intensities, as well as vestibular problems. A major long-term goal of ECochG is to help differentiate outer hair cell (OHC) from inner hair cell (IHC) or presynaptic losses, and from auditory nerve fiber (ANF) or postsynaptic losses, which are all presently lumped together as sensorineural hearing loss. Differential diagnosis of different forms of sensorineural hearing loss could prove useful in improving hearing aid fitting, in predicting cochlear implantation outcomes, and in individualized regenerative medicine (McLean et al., 2016, 2017).

ECochG measurements are believed to originate, in general, from at least four distinct cellular sources, the receptor potentials of OHCs and IHCs, and the dendritic potentials and spikes of ANFs. The phases or polarities of these components can vary along the cochlea in a complex fashion that depends on stimulus characteristics and electrode placement, confounding their separation and interpretation (Chertoff et al., 2012). For example, the origins of the commonly measured summating potential (SP) and cochlear microphonic (CM) are still unknown for the wide range 
of stimulus parameters and recording locations. The older term "cochlear response", which seems to have become passé, thus remains an adequate descriptor of ECoch G recordings as long as their origins remain elusive. A newer term with the same purpose appears to be the "total response" (e.g., McClellan et al., 2014). Continuing the progress toward untangling the different origins of ECochG measurements is essential to advance the clinical utility of ECochG (e.g., Forgues et al., 2014; Lichtenhan et al., 2014; Fontenot et al.).

The first ECochG measurements were obtained somewhat serendipitously by Wever and Bray (1930), who were attempting to record from cat ANFs. Their alternating or AC potential would come to be known as the cochlear microphonic (CM) and its origin was attributed to the hair cells, primarily to the more numerous and sensitive OHCs (Dallos and Cheatham, 1976), which amplify and sharpen sound-induced vibrations before their detection by the sensory IHCs and ANFs. It was later discovered that ANF spiking could also contribute to $\mathrm{CM}$ measurements, particularly in response to lowerfrequency sounds $(<1-2 \mathrm{kHz})$, and that IHCs contributed as well (Eggermont, 1974; Chertoff et al., 2002; Lichtenhan et al., 2014). This blend of responses became known as the auditory nerve neurophonic (ANN, e.g., Snyder and Schreiner, 1984; Forgues et al., 2014), which is simply a cochlear response to intense, lowfrequency sounds. The Auditory Nerve Overlapped Waveform (ANOW; Lichtenhan et al., 2013, 2014) differs from the ANN in that it is evoked by low to moderate level sounds, and its cellular and spatial origins are known. ECochG measurements can be DC-biased by the summating potential (SP), and show compound action potential (CAP) responses to stimulus onsets and sometimes offsets, reflecting the synchronous spiking of ANFs (Davis et al., 1958; Ruben et al., 1961). The CAP is wave I of the auditory brainstem response (ABR), first characterized by Jewett and Williston (1971).

A long-standing use of ECochG has been to objectively corroborate a symptomatic and case-history diagnosis of endolymphatic hydrops in Meniere's disease and other pathological states (endolymphatic hydrops is not limited to Meniere's). In ears with endolymphatic hydrops, the SP/CAP ratio can be increased, due mainly to an increase in the SP, but also to a decrease in the CAP. Despite much research, it is not known whether the sensitivity and specificity of ECochG for detecting endolymphatic hydrops is high enough to be useful for individual patients. Sass (1998) reported high sensitivity and specificity (87 and 100\%, respectively) when transtympanic click and $1 \mathrm{kHz}$ tone burst SP/CAP ratios were combined with the increased CAP latency difference between rarefaction and condensation stimulus clicks that is also typical of ears with endolymphatic hydrops. Others have also reported good sensitivity and specificity by using the SP/CAP area (e.g., Ferraro, 2010). As reviewed by Eggermont and Hornibrook, the results of some other studies have been less encouraging, but there is consensus that tone burst ECochG presently yields the best results (Hornibrook). In a promising new approach, Lichtenhan et al.induced endolymphatic hydrops in guinea pigs using three classical manipulations and found that changes in the ANOW were more sensitive to small degrees of endolymphatic hydrops than were changes in traditional measures such as CAP thresholds and the endocochlear potential, suggesting that the ANOW could be useful in the early detection of endolymphatic hydrops.

ECochG can be used in the diagnosis of auditory neuropathy (Widen et al., 1995; Rance and Starr, 2015), an umbrella term that includes many etiologies such as drug- or hypoxiainduced IHC loss (Harrison, 1998; Salvi et al.), noise- and agerelated synaptopathy (Kujawa and Liberman, 2015), hereditary synaptopathy and neuropathy (e.g., mutations of OTOF, OPA1, and other genes; Santarelli et al., 2013), and even acoustic neuroma. While MRI can be useful in confirming some cases of auditory neuropathy (e.g., Roche et al., 2010), it is typically diagnosed when an absent or abnormal CAP or ABR, even at high stimulus levels, co-occurs with a robust $\mathrm{CM}$ and/or otoacoustic emissions (OAEs). Speech perception deficits, both in quiet and in noise, are worse than expected from the audiometric loss. Identifying ears with auditory neuropathy is important for predicting cochlear implant outcomes, which are generally poorer compared to non-neuropathic patients (McMahon et al., 2008; Walton et al., 2008; Harrison et al., 2015; Santarelli et al., 2015).

Salvi et al.provided an instructive review of selective IHC loss in chinchillas due to the cancer drug carboplatin. Substantial IHC loss had no measurable effect on OAEs or the CM (however, see Chertoff et al., 2002), but reduced SP and CAP amplitudes. Tone thresholds in quiet were unaffected by IHC losses of up to $80 \%$, but thresholds in noise were elevated (Lobarinas et al., 2016). Importantly, the chinchilla carboplatin studies reviewed by Salvi et al. were also among the first to provide compelling evidence for synaptic gain increases in the central auditory system in response to decreased peripheral input. While increased central gain can lead to improved audibility in quiet conditions (see e.g.,Hoben et al.), it might also lead to potentially bothersome tinnitus and hyperacusis (Noreña, 2011; Schaette and McAlpine, 2011; Pienkowski et al., 2014; Brotherton et al., 2015; Paul et al., 2017).

ECochG is a promising candidate for detecting noise- and age-related cochlear synaptopathy (Kujawa and Liberman, 2009, 2015; Sergeyenko et al., 2013). It was recently reported that college student musicians with normal audiometric thresholds up to $8 \mathrm{kHz}$, but mild hearing losses at $10-16 \mathrm{kHz}$, showed significantly increased click-evoked SP amplitudes and slightly decreased CAP amplitudes (Liberman et al., 2016), changes reminiscent of endolymphatic hydrops but in this case attributed to noise-induced synaptopathy. Bramhall et al. (2017) found reduced CAP amplitudes in military veterans with high noise exposure histories, and in non-veterans who reported a history of firearm use, compared with veterans and non-veterans with lower noise histories. Importantly, the reduced CAP amplitudes could not be explained by $\mathrm{OHC}$ dysfunction, as assessed with distortion product OAEs (DPOAEs). Other studies using CAP or ABR wave I amplitudes (as well as other metrics) have failed to detect evidence of synaptopathy in noise-exposed adults (e.g., Prendergast et al., 2017). However, it may be that people who regularly subject themselves to high recreational noise doses do so because of their "tougher" ears, which sustain less damage 
than the potentially more "tender" ears of people who avoid loud music and noise (see e.g., Henderson et al., 1993 for a general discussion of this issue).

Grinn et al. reported CAP and DPOAE amplitudes, and Words-in-Noise (WIN) performance in a group of young adults before, and 1 and 7 days after a loud recreational event, typically a concert (average dose of $93 \mathrm{~dB} A$ for $4 \mathrm{~h}$, range 73-104 dB A for $1.5-16 \mathrm{~h}$ ). Consistent with the notion of tough vs. tender ears, there was no correlation between the noise dose and the amount of temporary threshold shift (TTS) measured across study participants. Most showed a 1 day TTS of $<10 \mathrm{~dB}$ (with full recovery at 7 days), accompanied by correspondingly small but significant temporary decreases in WIN scores. DPOAE amplitudes were affected at 1 day but only at $6 \mathrm{kHz}$, whereas CAP amplitudes to clicks and $2-4 \mathrm{kHz}$ tone bursts were not affected. These results argue against the development of synaptopathy after a single recreational noise dose, consistent with laboratory noise exposure that caused a TTS in humans (Lichtenhan and Chertoff, 2008). It is likely that a number of such exposures is needed to produce permanent damage in primates (Pienkowski, 2017; Valero et al., 2017).

To reduce the prevalence of noise-induced hearing loss, tinnitus, and hyperacusis, it would be helpful to identify those with especially tender ears. Maison and Liberman (2000) showed that the strength of the medial olivocochlear (MOC) efferent reflex in guinea pigs, as measured by the contralateral suppression of DPOAEs, was strongly correlated with lower TTS after acoustic trauma. Unfortunately, this finding has yet to be replicated in humans (e.g., Hannah et al., 2014). Smith et al.made measurements of chirp-evoked human CAPs, confirming the original finding that chirps yield larger CAP amplitudes than clicks (Chertoff et al., 2010). Smith et al. found that CAP amplitudes were more strongly contralaterally suppressible than were DPOAE amplitudes, similar to the results of previous animal and human studies (Puria et al., 1996; Lichtenhan et al., 2016). Verschooten et al. made progress in studying the human MOC reflex triggered by ipsilateral sound, by proposing how to separate MOC effects from the confounds of mechanical and neural masking.

This Research Topic reports innovations in recording techniques and signal processing that point to new potentially useful roles for ECochG in clinical practice (Charaziak et al.; Cook et al.; Kennedy et al.). Other innovations have noteworthy applications associated with cochlear implantation. Bester et al., Dalbert et al., Koka et al., and O'Connell et al., used ECochG to objectively assess residual, low-frequency acoustic hearing in ears implanted with hybrid electric-acoustic stimulation devices. $\mathrm{He}$ et al. comprehensively reviewed the electrically-evoked CAP

\section{REFERENCES}

Bramhall, N. F., Konrad-Martin, D., McMillan, G. P., and Griest, S. E. (2017). Auditory brainstem response altered in humans with noise exposure despite normal outer hair cell function. Ear Hear. 38, e1-e12. doi: 10.1097/AUD.0000000000000370 or eCAP, including its applications in establishing implant candidacy, in intraoperative monitoring for electrode guidance, and in post-operative device programming and outcome assessment. Riggs et al. made intraoperative measurements from child and adult implantees with and without diagnosed auditory neuropathy, and found results consistent with better hair cell but poorer neural function compared to non-neuropathic patients. While it remains a challenge to accurately estimate ANF survival in implant candidates, Pardo-Jadue et al. suggest that tymptrode measurements of spontaneous ANF firing (in the absence of sound or other stimulation) could be helpful in this regard.

The telemetric innovations of modern cochlear implants have advanced research in intracochlear ECochG. Kim et al. reported the first intracochlear ECochG measurements from cochlear implant (Nucleus Hybrid L24) users. Koka and Litvak performed the first intracochlear ECochG recordings in response to simultaneous electrical and acoustic stimulation in patients implanted with Advanced Bionics HiRes 90K Advantage. The results of these pioneering measurements may point the way forward to objectively programming hybrid cochlear implants and better predicting speech outcomes.

The past informs the present, as the saying goes, and this is certainly true of the field of ECochG. It is usual for even good data to be misinterpreted in the context of the available theories of the day. Likewise, it is usual for previous interpretations to become outdated as new advances are made. Nevertheless, interpretations, not data, are typically the main intellectual drive of textbooks and review articles, and new trainees to a field often begin with these sources. Once a knowledge base becomes firmly entrenched, it can sometimes be difficult and uncomfortable to realize that a framework is no longer adequate to encapsulate new findings, and needs updating. We hope to have clarified some of the main ideas, terminology, and origins of ECochG measurements, and encourage all to study the almost 90 year history of this field.

\section{AUTHOR CONTRIBUTIONS}

MP: drafted the manuscript; JL and MP: edited the manuscript; JL: organized the Research Topic; JL, OA, and MP: shared editing responsibilities on the Research Topic.

\section{ACKNOWLEDGMENTS}

We thank professors Jos J. Eggermont, James W. Hall III, and John J. Guinan Jr. for their productive comments on earlier drafts of this manuscript. We also thank professor Christoph Schreiner for helpful discussion on this work. 
Chertoff, M. E., Earl, B. R., Diaz, F. J., and Sorensen, J. L. (2012). Analysis of the cochlear microphonic to a low-frequency tone embedded in filtered noise. J. Acoust. Soc. Am. 132, 3351-3362. doi: 10.1121/1. 4757746

Chertoff, M. E., Lichtenhan, and, J., and Willis, M. (2010). Click- and chirpevoked human compound action potentials. J Acoust Soc Am. 127, 2992-2996. doi: $10.1121 / 1.2967890$

Dallos, P., and Cheatham, M. A. (1976). Production of cochlear potentials by inner and outer hair cells. J. Acoust. Soc. Am. 60, 510-512. doi: 10.1121/1.381086

Davis, H., Deatherage, B. H., Eldredge, D. H., and Smith, C. A. (1958). Summating potentials of the cochlea. Am. J. Physiol. 195, 251-261.

Eggermont, J. J. (1974). Basic principles for electrocochleography. Acta. Otolaryngol. Suppl. 316, 7-16.

Ferraro, J. A. (1986). Electrocochleography. Semin. Hear. 7, 239-240.

Ferraro, J. A. (2010). Electrocochleography: a review of recording approaches, clinical applications, and new findings in adults and children. J. Am. Acad. Audiol. 21, 145-152. doi: 10.3766/jaaa.21.3.2

Ferraro, J. A., and Ferguson, R. (1989). Tympanic ECochG and conventional ABR: a combined approach for the identification of wave I and the I-V interwave interval. Ear. Hear. 10, 161-166.

Forgues, M., Koehn, H. A., Dunnon, A. K., Pulver, S. H., Buchman, C. A., Adunka, O. F., et al. (2014). Distinguishing hair cell from neural potentials recorded at the round window. J. Neurophysiol. 111, 580-593. doi: 10.1152/jn.00446. 2013

Hannah, K., Ingeborg, D., Leen, M., Annelies, B., Birgit, P., Freya, S., et al. (2014). Evaluation of the olivocochlear efferent reflex strength in the susceptibility to temporary hearing deterioration after music exposure in young adults. Noise Health 16, 108-115. doi: 10.4103/1463-1741. 132094

Harrison, R. V. (1998). An animal model of auditory neuropathy. Ear. Hear. 19, 355-361.

Harrison, R. V., Gordon, K. A., Papsin, B. C., Negandhi, J., and James, A. L. (2015). Auditory neuropathy spectrum disorder (ANSD) and cochlear implantation. Int. J. Pediatr. Otorhinolaryngol. 79, 1980-1987. doi: 10.1016/j.ijporl.2015.10.006.

Henderson, D., Subramaniam, M., and Boettcher, F. A. (1993). Individual susceptibility to noise-induced hearing loss: an old topic revisited. Ear. Hear. $14,152-168$.

Jewett, D. L., and Williston, J. S. (1971). Auditory-evoked far fields averaged from the scalp of humans. Brain 94, 681-696.

Kujawa, S. G., and Liberman, M. C. (2009). Adding insult to injury: cochlear nerve degeneration after "temporary" noise-induced hearing loss. J. Neurosci. 29, 14077-14085. doi: 10.1523/JNEUROSCI.2845-09.2009

Kujawa, S. G., and Liberman, M. C. (2015). Synaptopathy in the noiseexposed and aging cochlea: primary neural degeneration in acquired sensorineural hearing loss. Hear. Res. 330, 191-199. doi: 10.1016/j.heares.2015. 02.009

Liberman, M. C., Epstein, M. J., Cleveland, S. S., Wang, H., and Maison, S. F. (2016). Toward a differential diagnosis of hidden hearing loss in humans. PLoS ONE 11:e0162726. doi: 10.1371/journal.pone.0162726

Lichtenhan, J. T., and Chertoff, M. E. (2008). Temporary hearing loss influences post-stimulus time histogram and single neuron action potential estimates from human compound action potentials. J. Acoust. Soc. Am. 123, 2200-2212. doi: $10.1121 / 1.2885748$

Lichtenhan, J. T., Cooper, N. P., and Guinan, J. J. Jr. (2013). A new auditory threshold estimation technique for low frequencies: proof of concept. Ear. Hear. 34, 42-51. doi: 10.1097/AUD.0b013e31825f9bd3

Lichtenhan, J. T., Hartsock, J. J., Gill, R. M., Guinan, J. J. Jr., and Salt, A. N. (2014). The auditory nerve overlapped waveform (ANOW) originates in the cochlear apex. J. Assoc. Res. Otolaryngol. 15, 395-411. doi: 10.1007/s10162-0140447-y

Lichtenhan, J. T., Wilson, U. S., Hancock, K. E., and Guinan, J. J. Jr. (2016). Medial olivocochlear efferent reflex inhibition of human cochlear nerve responses. Hear. Res. 333, 216-224. doi: 10.3109/14992027.2015.1122238

Lobarinas, E., Salvi, R., and Ding, D. (2016). Selective inner hair cell dysfunction in chinchillas impairs hearing-in-noise in the absence of outer hair cell loss. J. Assoc. Res. Otolaryngol. 17, 89-101. doi: 10.1007/s10162-0150550-8
Maison, S. F., and Liberman, M. C. (2000). Predicting vulnerability to acoustic injury with a noninvasive assay of olivocochlear reflex strength. J. Neurosci. 20, 4701-4707. doi: 10.1523/JNEUROSCI.20-12-04701.2000

McClellan, J. H., Formeister, E. J., Merwin, W. H. III, Dillon, M. T., Calloway, N., Iseli, C., et al. (2014). Round window electrocochleography and speech perception outcomes in adult cochlear implant subjects: comparison with audiometric and biographical information. Otol. Neurotol. 35, e245-e252. doi: 10.1097/MAO.0000000000000557

McLean, W. J., McLean, D. T., Eatock, R. A., and Edge, A. S. (2016). Distinct capacity for differentiation to inner ear cell types by progenitor cells of the cochlea and vestibular organs. Development 143, 4381-4393. doi: $10.1242 / \operatorname{dev} .139840$

McLean, W. J., Yin, X., Lu, L., Lenz, D. R., McLean, D., Langer, R., et al. (2017). Clonal expansion of Lgr5-positive cells from mammalian cochlea and high-purity generation of sensory hair cells. Cell. Rep. 18, 1917-1929. doi: 10.1016/j.celrep.2017.01.066

McMahon, C. M., Patuzzi, R. B., Gibson, W. P., and Sanli, H. (2008). Frequency-specific electrocochleography indicates that presynaptic and postsynaptic mechanisms of auditory neuropathy exist. Ear. Hear. 29, 314-325. doi: 10.1097/AUD.0b013e3181662c2a

Noreña, A. J. (2011). An integrative model of tinnitus based on a central gain controlling neural sensitivity. Neurosci. Biobehav. Rev. 35, 1089-1109. doi: 10.1016/j.neubiorev.2010.11.003

Paul, B. T., Bruce, I. C., and Roberts, L. E. (2017). Evidence that hidden hearing loss underlies amplitude modulation encoding deficits in individuals with and without tinnitus. Hear. Res. 344, 170-182. doi: 10.1016/j.heares.2016. 11.010

Pienkowski, M. (2017). On the etiology of listening difficulties in noise despite clinically normal audiograms. Ear. Hear. 38, 135-148. doi: 10.1097/AUD.0000000000000388

Pienkowski, M., Tyler, R. S., Roncancio, E. R., Jun, H. J., Brozoski, T., Dauman, N., et al. (2014). A review of hyperacusis and future directions: part II. Measurement, mechanisms, and treatment. Am. J. Audiol. 23, 420-436. doi: 10.1044/2014_AJA-13-0037

Prendergast, G., Guest, H., Munro, K. J., Kluk, K., Léger, A., Hall, D. A., et al. (2017). Effects of noise exposure on young adults with normal audiograms I: Electrophysiology. Hear. Res. 344, 68-81. doi: 10.1016/j.heares.2016.10.028

Puria, S., Guinan, J. J. Jr., and Liberman, M. C. (1996). Olivocochlear reflex assays: effects of contralateral sound on compound action potentials versus ear-canal distortion products. J. Acoust. Soc. Am. 99, 500-507.

Rance, G., and Starr, A. (2015). Pathophysiological mechanisms and functional hearing consequences of auditory neuropathy. Brain 138, 3141-3158. doi: 10.1093/brain/awv270

Roche, J. P., Huang, B. Y., Castillo, M., Bassim, M. K., Adunka, O. F., and Buchman, C. A. (2010). Imaging characteristics of children with auditory neuropathy spectrum disorder. Otol. Neurotol. 31, 780-788.

Ruben, R. J., Bordley, J. E., and Lieberman, A. T. (1961). Cochlear potentials in man. Laryngoscope 71, 1141-1164. doi: 10.1288/00005537-196110000-00001

Ruth, R. A., Lambert, P. R., and Ferraro, J. A. (1988). Electrocochleography: methods and clinical applications. Am. J. Otol. Suppl, 9, 1-11.

Santarelli, R., del Castillo, I., and Starr, A. (2013). Auditory neuropathies and electrocochleography. Hear. Bal. Commun. 11, 130-137. doi: 10.3109/21695717.2013.815446

Santarelli, R., Rossi, R., Scimemi, P., Cama, E., Valentino, M. L., LaMorgia, C., et al. (2015). OPA1-related auditory neuropathy: site of lesion and outcome of cochlear implantation. Brain 138, 563-576. doi: 10.1093/brain/awu378

Sass, K. (1998). Sensitivity and specificity of transtympanic electrocochleography in Ménière's disease. Acta Otolaryngol. 118, 150-156. doi: 10.1080/00016489850154838

Schaette, R., and McAlpine, D. (2011). Tinnitus with a normal audiogram: physiological evidence for hidden hearing loss and computational model. $J$. Neurosci. 31, 13452-13457. doi: 10.1523/JNEUROSCI.2156-11.2011

Sergeyenko, Y., Lall, K., Liberman, M. C., and Kujawa, S. G. (2013). Age-related cochlear synaptopathy: an early-onset contributor to auditory functional decline. J. Neurosci. 33, 13686-13694. doi: 10.1523/JNEUROSCI.178313.2013

Snyder, R. L., and Schreiner, C. E. (1984). The auditory neurophonic: basic properties. Hear. Res. 15, 261-280. 
Valero, M. D., Burton, J. A., Hauser, S. N., Hackett, T. A., Ramachandran, R., and Liberman, M. C. (2017). Noise-induced cochlear synaptopathy in rhesus monkeys (Macaca mulatta). Hear. Res. 353, 213-223. doi: 10.1016/j.heares.2017.07.003

Walton, J., Gibson, W. P., Sanli, H., and Prelog, K. (2008). Predicting cochlear implant outcomes in children with auditory neuropathy. Otol. Neurotol. 29, 302-309. doi: 10.1097/MAO.0b013e318164d0f6

Wever, E. G., and Bray, C. W. (1930). Auditory nerve impulses. Science 71, 215. doi: $10.1126 /$ science.71.1834.215

Widen, J. E., Ferraro, J. A., and Trouba, S. E. (1995). Progressive neural hearing impairment: case report. J. Am. Acad. Audiol. 6, 217-224.
Conflict of Interest Statement: The authors declare that the research was conducted in the absence of any commercial or financial relationships that could be construed as a potential conflict of interest.

Copyright (๑) 2018 Pienkowski, Adunka and Lichtenhan. This is an open-access article distributed under the terms of the Creative Commons Attribution License (CC $B Y)$. The use, distribution or reproduction in other forums is permitted, provided the original author(s) and the copyright owner are credited and that the original publication in this journal is cited, in accordance with accepted academic practice. No use, distribution or reproduction is permitted which does not comply with these terms. 